\title{
Temporal Deployment of Attention by Mental Training: an fMRI Study
}

\author{
Souhir Daly ${ }^{1}$ - Jade Thai ${ }^{2}$ - Chama Belkhiria ${ }^{1}$ - Chistelle Langley ${ }^{2}$ - Alain Le Blanche ${ }^{1,3}$ - Giovanni de Marco ${ }^{1,4}$
}

(C) The Psychonomic Society, Inc. 2020, corrected publication 2021

\begin{abstract}
In this study, we employed a visuo-motor imagery task of alertness as a mental training to examine temporal processing of motor responses within healthy young adults. Participants were divided into two groups (group $1 ; \mathrm{n}=20$ who performed the mental training before the real physical task and a control group who performed the physical task without mental training). We vary the time interval between the imperative stimulus and the preceding one (fore-period) in which temporal preparation and arousal increase briefly. Our behavioural results provide clear evidence that mental training reinforces both temporal preparation and arousal, by shortening reaction time (RT), especially for the shortest fore-periods (FP) within exogenous "FP $250 \mathrm{ms"}(p=0.008)$ and endogenous alertness "FP $650 \mathrm{~ms}$ " $(p=0.001)$. We investigated how the brain controls such small temporal changes. We focus our neural hypothesis on three brain regions: anterior insula, dorsolateral prefrontal cortex, and anterior cingulate cortex and three putative circuits: one top-down (from dorsolateral prefrontal cortex to anterior cingulate cortex) and two bottom-up (from anterior insula to dorsolateral prefrontal cortex and anterior cingulate cortex). In fMRI, effective connectivity is strengthened during exogenous alertness between anterior insula and dorsolateral prefrontal cortex $(p=0.001)$, between anterior insula and cingulate cortex $(p=0.01)$, and during endogenous alertness between dorsolateral prefrontal cortex and anterior cingulate cortex $(p=0.05)$. We suggest that attentional reinforcement induced by an intensive and short session of mental training induces a temporal deployment of attention and allow optimizing the time pressure by maintaining a high state of arousal and ameliorating temporal preparation.
\end{abstract}

Keywords Attention $\cdot$ Arousal $\cdot$ Mental training $\cdot$ Time pressure $\cdot$ Connectivity

Accurate timing of future events is a fundamental ability that we routinely apply in various activities that our environment

This work was partly supported by LINP2 (Interdisciplinary Laboratory in Neurosciences, Physiology and Psychology), Université Paris Nanterre, F92000 Nanterre, France and the COMUE University Paris Lumière (UPL).

Souhir Daly

souhir.daly91@gmail.com

1 LINP2 (Interdisciplinary Laboratory in Neurosciences, Physiology and Psychology), Université Paris Nanterre, 200 Avenue de la république, 92000 Nanterre, France

2 Clinical Research \& Imaging Centre (CRICBristol), Bristol Medical School, Faculty of Health Sciences, University of Bristol, Bristol, UK

3 Hôpital René-Dubos de Pontoise and Université de Versailles-Saint-Quentin, Simone Veil UFR des Sciences de la Santé, Montigny-le-Bretonneux, France

4 COMUE Paris Lumières University, Paris, France continuously provides and in which we make use of temporal strategies and eventualities that we have learned during our lives and that we update depending on new circumstances (Los et al. 2014). The failure in anticipating and rapidly orienting attention at a giving point may constitute a real problem that can negatively affect decisions and our daily lives. Johnson and Raab (2003) show that among experts, the strategies that generate the least options (treatment) are the best and produce more consistent decisions. The Naturalistic Decision Making community talks about "intuition" as an expression of experience as people build up patterns that enable them to rapidly size up situations and make rapid decisions without having to compare options. (Klein et al. 2010; Kahneman \& Klein 2009) have argued that intuition can only be trusted if it reflects repeated experiences and allow making relevant decisions despite high time pressure. In this context, we propose to investigate temporal processing mechanisms using the alertness concept and we raise the possibility that temporal features of alertness could serve as biomarker of temporal processing and mental effort. 
Neuroscientific and psychological studies in Functional MRI (fMRI) show that alertness is a fundamental component of attention that mobilizes vigilance and time expectancy (temporal preparation) of an upcoming event (Sturm et al., 1999; Sturm \& Willmes, 2001; Clemens et al., 2011; Posner \& Bois, 1971). Dye, Green, and Bavelier (2009) define alertness as "the ability to make use of a cue which provides information about the onset time of a target stimulus, and thus triggers the allocation of attention at a given point in time." It includes two fundamental components; endogenous and exogenous alertness. Exogenous alertness is stimulus-driven, automatically activated by the sudden onset of a stimulus and drawn towards a stimulus in the visual field. It is supported by a regulatory bottom-up control system and reflects more involuntary attention and automatic processes. Conversely, endogenous alertness is conceptually driven, voluntarily allocated to a location in the visual field usually by interpreting a cue that directs one to the target, and can be sustained for several seconds. It represents higher cognitive functions supported by reflexive analytical top-down control system (Barbot, Landy, \& Carrasco, 2012; Carrasco, 2011; Posner, Cohen, \& Rafal, 1982). Therefore, our study was designed to investigate the neural circuits that are responsible for both top-down and bottom-up processes that control temporal processing.

Herein, we seek to elucidate the neural circuits that participate in the deployment of attention in a context of temporal optimization of the action (preparation) and also in a more intuitive way the processes that may facilitate (accelerate) stages of information processing (Nobre \& Coull, 2010). More specifically, we try to demonstrate that if the participants are mentally and intensively trained to react to target stimulus using a motor imagery task, we can increase the vigilance level (arousal) and improve the temporal preparation of action. In fact, motor imagery (MI) corresponds to an active process in which the human body is involved and during which the representation of a specific action is reproduced internally within working memory without any overt motor output (Jackson et al., 2001). It can be realized with different modalities: visual MI, which requires individuals to self-visualize the execution of the movement either from a first (internal visual imagery) or third-person (external visual imagery) perspective, or kinesthetic MI, which requires individuals to "feel the movement," to perceive the sensations normally associated with its execution, such as muscle stretching and contractions (Guillot et al., 2009). When the act of imaging a movement is repeated a few times, we talk about "mental practice" or "mental training," which consists of a training method by which the internal reproduction of a given motor act is repeated extensively with the intention of initiating, learning a new ability or improving a known skill (Decety \& Grèzes, 1999; Jackson, Lafleur, Malouin, Richards, \& Doyon, 2001).
The motor inhibition helps participants to focus more on the cognitive component of the task rather than the physical aspect (execution) to increase concentration and attention. Also, the internal imagined images may stimulate kinesthetic feeling of movements and activate automatically different mechanisms and processes involved in the initiation of movements during motor task (Jackson et al., 2001). Our research team published different papers (in biomechanical and fMRI) in developing paradigms of motor (initiation) preparation to better understand the gait initiation and postural instability in certain neurodegenerative diseases: e.g., amyotrophic lateral sclerosis (ALS) (Abidi et al., 2020; Feron et al., 2018). We believe that attention may play a determining role in initiating movement. This is the reason why we have chosen this "mental imagery training" task, which should reinforce the attentional control in ALS and multiple sclerosis "MS" patients. In MS, the question could concern the motor anticipation, which is altered by the fatigue, very strongly felt in this population. In ALS, the question concerns more the initiation of the movement, but also probably anticipation in relation to the pain. So, using motor imagery repetitively represents a mental training that cognitively helps to optimize performance by stimulating working memory, increasing vigilance, and physically by improving motor execution. It is a method through which performance can be facilitated and improved (Decety \& Grèzes, 1999; Jackson et al., 2001).

In addition to MI, we manipulate the so called "fore-period" (FP) as a behavioral indictor of temporal processing. It represents, according to (Posner \& Bois 1971), a vigilance situation where alertness must be mobilized rapidly and maintained over a relatively brief interval. It represents the waiting time interval between the imperative stimulus requiring response and a preceding one either a warning stimulus (endogenous alertness) or an accessory stimulus (Hackley et al., 2009; Stahl \& Rammsayer, 2005) preceding the imperative stimulus (exogenous alertness) (Périn et al., 2010; Vallesi \& Shallice, 2007). In other words, the FP represents a short time delay which helps to optimize an upcoming response. It includes the capacity of anticipating future events and reducing uncertainty about them (Brunia, De Jong, Van Den BergLenssen, \& Paans, 2000). The ability to estimate exactly the time of stimulus appearing, as time goes on, depends on the FP duration.

By varying different short FP time lengths and by defining specific brain regions, we can elucidate the way in which the brain controls attention following short temporal changes. We previously demonstrated in Périn et al. (2010) the neural substrates of alertness involved key brain regions, including especially the dorsolateral prefrontal cortex (DLPFC) and anterior cingulum cortex (ACC). Recently, others studies (Coste \& Kleinschmidt, 2016; Sadaghiani \& D’Esposito, 2015) have demonstrated that alertness is a fundamental function of the Cingulo-operculum network in which anterior insula (AI) and 
anterior cingulate play a crucial role in the maintenance of exogenous alertness. Herein, our study will focus on a specific network including three brain regions: anterior insula (AI), DLPFC, and ACC, known to control attention during cognitively demanding tasks (Cauda et al., 2011). Anterior insula has been demonstrated to intervene in the arousal regulation during alertness tasks (Craig, 2002; Critchley, Melmed, Featherstone, Mathias, \& Dolan, 2002); it has been associated with a bottom-up capture of focal attention particularly during exogenous attention (Han et al., 2018). More specifically, AI feeds the ACC with the necessary information, which in turn spreads out these signals to the cortical regions. It, therefore, has a powerful causal influence on the ACC, which facilitates selection and motor response preparation; "AI-ACC" circuit moderates arousal (Sridharan, Levitin, \& Menon, 2008) when the demand of exogenous control of attention increases. Also, it detects salient events and generates transient signals to alert the executive network (e.g., DLPFC) to maintain vigilance. In addition to that, $\mathrm{AI}$ is known to play a crucial role in time perception; it uses the representation of body states to perceive time (Craig, 2009). By temporally integrating transient signals from the body, it helps to produce "critical moments" and increase the perception of duration. The DLPFC, known as the locus of temporal readiness, has been found to be strongly active during fore-periods or "delay periods," increases predictability of target occurrence, and cognitively prepares for the coming action during alertness tasks. The ACC controls arousal and prepare the motor output; it helps to energize and facilitate temporal processing during brief delay periods (FPs) (Niemi \& Näätänen, 1981).

Many fMRI studies on attention have tried to understand "where" and "how" attention is controlled in the brain using different components of attention (Clemens et al., 2011, 2013; Mottaghy et al., 2006; Périn et al., 2010; W. Sturm et al., 1999); however, no study has focused on the attentional control reinforcement after mental training. Therefore, we investigated the hypothesis that alertness (after mental training) will reinforce dynamically (from a neural model of psychophysiological interaction) both top-down (endogenous alertness) and bottom-up (exogenous alertness) circuits by modifying the cortical excitability in specific brain regions responsible for attentional control. In the model of effective connectivity proposed, AI, ACC, and DLPFC regions will constitute hub regions in the control of alertness, particularly in attentional control and timing aspects. We postulate that anterior insula may facilitate the integration of the temporal processing of the information by providing transient control signals over time, improving the perception of durations. Using the mental training as a reinforcement task of attentional control and manipulating FPs as temporal indicators of information processing would help our understanding of how these circuits underpin the temporal deployment of attention and participate in the transfer of information.

\section{Method}

\section{Participants}

This study was approved by the institutional research board of the CPP Ile-de-France Cergy-Pontoise. Thirty-nine healthy participants ( 20 females and 19 males) aged between 21 and 35 years $($ mean $=27.5$; standard deviation $[\mathrm{SD}]=$ 4.50) with no known history of neurological, psychiatric, or sensorimotor disorders were recruited. All participants were right-handed, as assessed by the Edinburgh inventory for right-handedness. In this study, we compared two groups: a group named wMT "with mental training" who did the visuo-motor imagery before the real physical task $(\mathrm{n}=19)$, and a group named "woMT "without mental training" $(\mathrm{n}=20)$.

\section{Neuropsychological assessment}

The main hypothesis of this study is that mental training (MT) would reinforce attention and improve or accelerate temporal processing. Based on this assumption, we have selected three attentional tests: the $\mathrm{d} 2$ test, Stroop test, and Trail-Making test, which may help to assess executive functions, in particular processing speed capacity (Bowie \& Harvey, 2006; Denney \& Lynch, 2009; Hsieh, Chen, Wang, \& Lai, 2008; Wassenberg et al., 2008). Knowing that temporal processing of information is directly related to speed processing and could optimize the dynamics of perceptual processing speed resulting in faster and more accurate processing of targets (Capizzi, Sanabria, \& Correa, 2012; Vangkilde, Coull, \& Bundesen, 2012), we compared performances before and after mental training to test our hypothesis and to assess the beneficial effect of MT. Therefore, both groups (wMT and woMT) underwent this neuropsychological battery. Concentration and speed capacities (sustained attention and visual scanning respectively) were measured with the $\mathrm{d} 2$ test (Bates \& Lemay, 2004); the score measured with $\mathrm{d} 2$ represents the percentage of errors calculated following this formulae $\mathrm{F} \%=(100 * \mathrm{~F}) / \mathrm{GZ}$ where $F$ is the number of errors (number of omissions + number of "d" that should not have been circled) and GZ is the number of circled items (Steinborn, Langner, Flehmig, \& Huestegge, 2018). Executive functions, selective attention, and cognitive inhibition were measured with the Stroop test (Words and Color) (Golden, 1976). The time to read colors names printed in incongruent colored ink has been measured. The trail-making test "TMT" (parts A \& B) is different from the Stroop test in the way of accomplishment and in terms of evaluated cognitive functions. While the Stroop test assesses selective attention and cognitive inhibition, the TMT provides information on visual search, scanning, and mental flexibility (Pena-Casanova et al., 2009; Tombaugh, 2004). 
Part A requires the participant to draw lines on a page connecting 25 numbers consecutively as quickly as possible. In Part B, the participant must draw lines alternating between numbers and letters in consecutive order. The score on each part represents the amount of time (in seconds) required to join in ascending order numbers (part A) and numbers + letters (part B) (Corrigan \& Hinkeldey, 1987; Gaudino, Geisler, \& Squires, 1995; Golden, 1976). All of the participants had to complete the three tests before and after the real physical task. Scores were compared using the $t$ test within each group.

\section{Behavioral assessment}

The experimental fMRI task was assessed with a block design. Twelve blocks were constructed within three conditions (sensorimotor control condition (T1), exogenous condition (T2), and endogenous condition (T3)). The order of the blocks was randomized through the whole task [T3-T1T2-T2-T1-T3-T3-T1-T2-T2-T1-T3]. RT trials were extracted from (T3) endogenous alertness and (T2) exogenous alertness. Twenty-one RT trials were extracted from each condition ( 7 trials for each level of force) and 168 trials from the whole task by subject.

The paradigm consists of two alertness conditions in which participants were asked to focus their attention and squeeze an handgrip with the left hand as quickly as possible when a target appeared (white square) respecting the required force level (low, medium, and high). The purpose of using the left hand is to incite participants to produce more mental effort during mental training. The three levels of forces are chosen to make the task less monotonous, requiring participants to prepare for the task without producing reflex (automatic) responses and to stay focused on the whole duration of the task.

During the exogenous alertness condition (T2), participants have no information about the occurrence of the imperative stimulus (target). In the endogenous alertness condition (T3), a black screen was presented to act as a temporal cue before the target. An instruction was displayed before each condition: "Squeeze the handgrip as quick as possible when a white square appears on the screen respecting the required force level (low, medium, and high) and without moving the head." In both conditions (exogenous and endogenous), each trial included a central fixation cross on a black screen ( 5 degrees visual field) (Figure 1). The target was displayed with different randomized fore-periods (FPs). The FP represents, according to Posner and Bois (1971), a vigilance situation where alertness must be increased rapidly and maintained over a relatively brief interval. In more specific terms, it is considered as the waiting time interval varying within a fixed range of values, which allows the subject to install a preparation (during exogenous alertness) and the preparatory interval between a warning cue and the imperative stimulus (during endogenous alertness). Durations between the temporal cue (black screen) and the target (white square) for endogenous condition were: 650,680 , and $710 \mathrm{~ms}$. The choice of these values ranging in 500-1,000 $\mathrm{ms}$ scale is shown in the literature to be an optimal time range for obtaining temporal preparation effect, which gradually decreases beyond this time interval (Olivier and Rival, 2002; Posner \& Rafal, 1987). Durations between the target onset of a trial and the preceding one for exogenous condition were 250, 280, and $310 \mathrm{~ms}$. In fact, exogenous alertness is often assessed in the literature with tasks with no informative cues, because it does not engage a real process of temporal preparation but accessory stimuli (with FP lower than $500 \mathrm{~ms}$ ) that could be used to engage a mechanism of implicit expectation. In our experiment, we have fixed FPs durations during this task on a temporal scale under $500 \mathrm{~ms}$ to account for a potential arousal modulation during the implicit temporal preparation of the target implemented over time. We have chosen the same FPs $(250,280$, and $310 \mathrm{~ms})$ for the exogenous alertness task so that the measures were comparable in time of occurrence of the imperative stimulus with the endogenous alertness task. We do not have any empirical evidence for the durations of the exogenous FPs. However, we realized that an objective marker of the autonomous nervous system, such as heart rate variability (HRV), could allow to quantify the influence of the ANS on the exogenous alertness, which is known to be involved in the activation of sympathetic pathways that change rapidly and automatically with homeostatic changes. Also, it is implicated to respond to unexpected stimuli and urgent behaviors (Craig, 2005, Rogers \& Andrew, 2002; Wittling, 1995; Jones, 2003; Robinson and Coyle, 1980). This neuro-anatomical architecture puts in premium the adaptive dimension of arousal as a function of brief time variations. In addition, physiologically, arousal changes have been shown to be related to changes in heart rate, pupil dilatation, respiratory rhythm, and galvanic-skin-response. All are physiological measures, which vary according to very short time scale. Taking together, it seems reasonable to adjust the durations of FPs on a time scale as close as possible to these physiological measurements, allowing for better characterization of arousal modulations under mental training.

A sensorimotor control (T1) task was used in which the participants had to perform a voluntary self-paced handgrip squeeze with a frequency around $1 \mathrm{~Hz}$ while watching passively a fast $(10 \mathrm{~Hz})$ flickering white square, avoiding synchronization with the squeezing. The visuo-motor imagery task was performed, as a mental training task, in the scanner by half of the participants before the real physical task (wMT group). They were told to execute exactly the same task but mentally by imaging squeezing the handgrip 


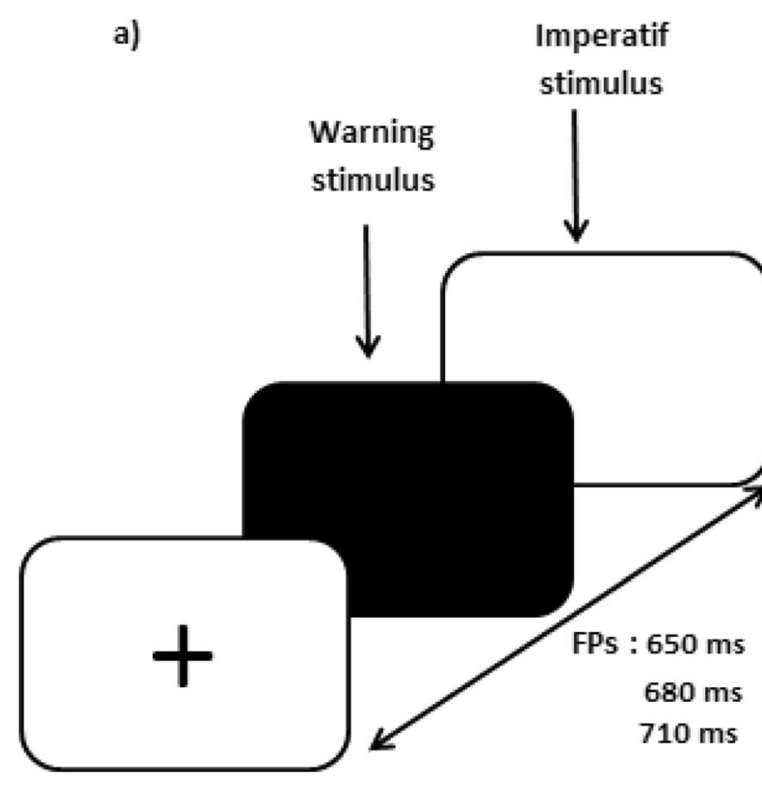

Fig. 1 (a) Endogenous condition: participants had to respond to a white square. The warning stimulus was displayed during $400 \mathrm{~ms}$ and preceded the imperative stimulus with a varying fore-periods "FPs." (b) Exogenous condition: participants had to respond to a white square. A central fixation b)

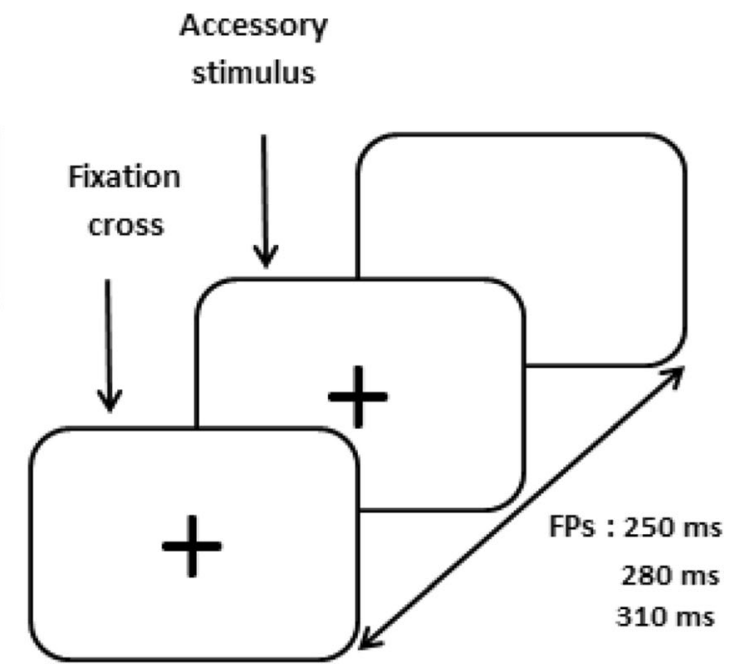

cross was displayed permanently. Instruction (concentrate and squeeze the handle with the required force level) was delivered at the beginning of each new condition (without motor output). Both tasks lasted 30 minutes $(2 \times 15$ minutes). The rest of participants did not perform the visuomotor imagery task (woMT group).

\section{RT and forces measurements}

Reaction time (RT) and force measures (low, medium, and high) recording were recorded by E-prime (version 2.0.8.22) and Acknowledge software (version 4.2) respectively. RTs were extracted for each trial and represent the interval duration between target onset and force amplitude onset. The RTs values measured were comparable to Périn et al. (2010) obtained when pressing a button. The force measures show significant differences between force levels (low vs. medium, low vs. high, and medium vs. high) in both groups, which shows that the participants correctly followed the force level instructions.

\section{MR data acquisition}

Neuroimaging data were acquired at Pontoise Hospital Centre on 1.5-Tesla, whole-body MRI system equipped with Sigma head volume coil (General Electric Medical System, Milwaukee, WI). Functional images with Blood-OxygenLevel Dependent (BOLD) contrast enhancement were acquired for each participant. Single-shot echo-planar images (EPIs) acquired using a typical $\mathrm{T} 2 *$-weighted gradient-echo sequence. A total of 310 EPI volumes $\mathrm{x} 2$ with no gap (TR/ $\mathrm{TE}=3,000 / 44 \mathrm{~ms}$; flip angle $=90$ degrees; matrix $=64 * 64$; FOV $=240 \times 240 \mathrm{~mm}^{2}$ ) were obtained for the experimental runs, whole brain volumes compromising 44 , axial slices with $3.75-\mathrm{mm}$ thickness (isotropic voxel volume $=52.7$ $\mathrm{mm}^{3}$ ). For structural images, conventional T1-weighed 3D imaging fast spoiled gradient recall — brain volume (FSPGR) BRAVO sequence was employed with these parameters, matrix: 256 x 256; flip angle: 12 degrees; TR/TE: $8.6 / 3.3 \mathrm{~ms}$; FOV: $240 \times 240 \mathrm{~mm}^{2} ; 168$ slices, $1-\mathrm{mm}^{3}$ isotropic voxel resolution.

\section{Image pre-processing}

Image pre-processing was performed using Statistical Parametric Mapping (SPM12) software (Wellcome Centre for Human Neuroimaging, University College London, UK; http://www.fil.ion.ucl.ac.uk/spm). EPI volumes were corrected to adjust for within-volume time differences and realigned with the first volume to correct for head movements. Functional scans were then co-registered with the 3D structural image and spatially normalized against the standard, stereotactic space of the Montreal Neurological Institute (MNI) template. Spatial smoothing was performed with 8-mm Gaussian kernel full width at the half maximum (FWHM). 


\section{SPM analyses}

BOLD hemodynamic responses were modelled as a box-car function convolved with a synthetic hemodynamic response function. The motion parameters were included in the General Linear Model (GLM) as regressors to correct the movement. A single-subject, fixed-effect model was created for each individual participant in order to perform the based-conditions random-effect analysis The results presented are from the image acquisition during performance of the real physical task with threshold set at $p<$ 0.05 , family-wise error (FWE) corrected for multiple comparisons. The sensorimotor condition has been used as control of the exogenous alertness: "Exogenous alertness condition > sensorimotor condition." The exogenous alertness has been used as control of the endogenous alertness: "endogenous alertness condition $>$ exogenous alertness condition." To ensure that all of the participants recruited the exogenous and endogenous alertness system regardless of mental training, a one-sample $t$ test of the full dataset $(\mathrm{n}=$ 39) was conducted. Then, we performed a two-sample $t$ test to compare the differences between the groups: "wMT" > "woMT."

\section{Psychophysiological interaction analysis}

We perform an effective connectivity analysis to examine explicitly the influence that one neural system exerts over another, either at a synaptic (i.e., synaptic efficacy) or at a population level (Friston et al., 1997). The effective connectivity must be understood as the experiment- and time-dependent, simplest possible circuit diagram that would replicate the observed timing relationships between recorded neurons. To examine the effective connectivity between the AI, ACC, and DLPFC regions, a psychophysiological interaction (PPI) analysis was performed to investigate interactions among these three brain regions. A PPI analysis tests the hypothesis that activity in one brain area can be explained by the interaction between the presence of a cognitive process (attentional reinforcement) and activity in another region of the brain. We predicted that the activity of a source region will covary with the activity of target regions. On the basis of anatomical connections, we performed an effective connectivity analysis to determine the effects of mental training on AI-ACC-DLPFC circuits. Through this analysis, we hypothesize that effective connectivity between "AI $\rightarrow$ DLPFC," "AI $\rightarrow$ ACC" (during exogenous alertness condition) and "DLPFC $\rightarrow$ ACC" (during endogenous condition), would increase after mental training. The AI was chosen as a seed (source) region in the exogenous alertness condition. In endogenous condition, DLPFC was chosen as seed region which exert a top down control on ACC.

\section{Statistical analyses}

The exclusionary criteria are related to the successful completion of the RT task. RT represents the temporal interval between the target onset (represented by a square) and the response onset. RTs less than $100 \mathrm{~ms}$ were considered as anticipations and were excluded from the analysis. Also, RTs above 1,000 ms were excluded, because they exceed the duration of the expected reaction times. Some omissions also were excluded when the participant does not respond to the imperative stimulus $(<1 \%$ only for the endogenous alertness, no omissions were observed in the exogenous alertness). By subject, 168 trials (endogenous and exogenous alertness) were extracted during the task. For the whole population, 6,720 trials were extracted. Less than $3.5 \%$ of the trials were excluded (230 RT trials) from all participants). Statistical analysis were performed in Statistica ${ }^{\circledR}$ software (version 13.3), and significance threshold was set to $p<0.05$. A two-way analysis of variance (ANOVA) groups ["wMT" vs "woMT"] x alertness conditions [exogenous vs. endogenous] was performed to examine effects of mental training on alertness. Then, another two-way ANOVA was conducted separately for each alertness condition with fore-periods [" 650 vs. 680 vs. 710 " for endogenous condition and " 250 vs. 280 vs. 310 " for exogenous condition] x groups ["wMT" vs. "woMT"] to examine effects of mental training on temporal processing. When the ANOVA indicated significant main effects and/or interactions, Bonferroni post-hoc comparisons were used to compensate for the family-wise-error in multiple comparisons for each group of pair wise comparisons. The observed power in all behavioral results was between $60 \%$ (interaction) and $90 \%$ (main factor). Size effects were also reported as Partial eta squared ( $\eta 2$ ) for the ANOVA analyses and Cohen's d for the $t$-tests (Cohen, 2013; Muller \& Cohen, 1989).

\section{Results}

\section{Neuropsychological results}

Comparing before and after the real physical task within "wMT" group, significant differences for the three cognitive tests were found: for $\mathrm{d} 2$ test (mean before mental training "F\%" = 6.27, $\mathrm{SD}=4.04$, mean after mental training "F\%" = 3.84, $\mathrm{SD}=3.46, p=0.009$ ), effect size: (Cohen's $d=0.6$ ); for Stroop test (mean before mental training $=81.41 \mathrm{~s}, \mathrm{SD}=$ 17.53, mean after mental training $=76.28 \mathrm{~s}, \mathrm{SD}=18.93, p=$ 0.005), effect size: (Cohen's $d=0.3$ ). Finally, with the TMT test, a significant difference was found for the second part of the test $($ part B) $($ mean before mental training $=49.37 \mathrm{~s}, \mathrm{SD}=$ 24.70, mean after mental training $=43.34 \mathrm{~s}, \mathrm{SD}=24.27, p=$ 0.02 ), effect size: (Cohen's $d=0.2$ ). No significant difference was observed within part A. Within the "woMT" group, no 
significant neuropsychological difference was found between before and after task completion.

\section{Behavioral results}

\section{Anticipations}

Anticipations have been measured within both groups in the whole task. Results of ANOVA [Groups x Alertness conditions] showed significant interaction [Groups $\mathrm{x}$ Alertness conditions $] F(1,2)=51.57 ; p=0.01$; effect size: partial $\eta^{2}$ $=0.9$. Post-hoc comparisons showed increased anticipation rate during endogenous condition compared with exogenous condition within each group; wMT group: endogenous condition: $11.69 \%$, exogenous condition: $1.93 \%, p=$ 0.003; woMT group: endogenous condition: $3.24 \%$, exogenous condition: $2.31 \%, p=0.01$. When comparing the endogenous condition between the two groups, the anticipation rate was significantly higher in the "wMT" group $(11.69 \%)$ than in the "woMT" group $(3.24 \% ; p=0.009)$, which confirms the acceleration of the response during the endogenous alertness after mental training No anticipation rate difference was found during the exogenous alertness.

\section{Reaction times}

Reaction times (RTs) have been measured within both groups during the whole task without separating FPs. ANOVA [Groups x Alertness conditions] revealed significant interaction [Groups $\times$ Alertness conditions] $F(1,23)=$ 12.46, $p=0.0004$; effect size: partial $\eta^{2}=0.03$. Post-hoc comparisons showed significant RTs differences between the two groups during both alertness conditions. For exogenous alertness condition, "woMT" group: mean $=400.28$, $\mathrm{SD}=116.61$, the $95 \%$ confidence interval (9CI) was [396.74; 409.15]; “wMT": mean = 379.29, SD = 101.30, $95 \% \mathrm{CI}=[375.87$; 388.11]) $p=0.0001$. For endogenous alertness condition; "woMT": mean $=388.53, \mathrm{SD}=130.07$, $95 \% \mathrm{CI}=[379.91 ; 395.13]$; “wMT" mean $=345.23, \mathrm{SD}=$ $137.37,95 \% \mathrm{CI}=[337.80 ; 352.82] p<10^{-5}$. RTs were significantly shorter within "wMT" group in both alertness conditions.

\section{Fore-periods}

RTs have been measured within both groups separating the FPs in the whole task (Figure 2).

Exogenous alertness condition ANOVA [Groups x FPs] showed significant interaction [Groups x FPs] $F(2,16)=$ $3.04, p=0.04$; effect size: partial $\eta^{2}=0.01$. Post-hoc comparisons showed within "woMT" group, significant differences of RTs between the shortest FP (250 ms) mean =
405.87, $\mathrm{SD}=129.52,95 \% \mathrm{CI}=[394.71 ; 417.02]$ and the longest FP $(310 \mathrm{~ms})$ mean $=384.09, \mathrm{SD}=99.33,95 \% \mathrm{CI}=$ [374.44; 393.74]; $p=0.001$ and between FP (280 ms) mean $=402.64, \mathrm{SD}=117.77,95 \% \mathrm{CI}=[392.72 ; 412.55]$ and $\mathrm{FP}$ (310 ms), $p=0.01$. No significant differences between FPs were observed in "wMT." When comparing each single fore-period between the two groups, post-hoc comparisons showed that RTs decreased significantly after mental training. Significant differences were found for shortest FP (250 $\mathrm{ms}$ ) between "woMT" and "wMT" mean $=380.97, \mathrm{SD}=$ $105.46,95 \% \mathrm{CI}=[370.33 ; 391.62], p=0.008$, and also for FP $(280 \mathrm{~ms})$ between "woMT and "wMT" mean $=374.15$, $\mathrm{SD}=89.85,95 \% \mathrm{CI}=[364.68 ; 383.61] ; p=0.001 . \mathrm{No}$ significant difference was found for the longest FP (310 $\mathrm{ms})$.

Endogenous alertness condition ANOVA [Groups x FPs] revealed significant interaction [Groups $\mathrm{xPP}$ ] $F(2,14)=3.1, p$ $=0.04$; effect size: partial $\eta^{2}=0.01$. Post-hoc comparisons showed within "woMT" group, a significant difference of RTs between the shortest FP (650 ms) mean $=415.37, \mathrm{SD}=$ $140.25,95 \% \mathrm{CI}=[401.77 ; 428.97]$ and the longest FP $(710$ ms) mean $=393.66, \mathrm{SD}=131.60,95 \% \mathrm{CI}=[380.85 ; 406.46]$; $p=0.01$. No significant difference was observed between FP 680 and $710 \mathrm{~ms}$. Within the "wMT" group, no significant difference was observed between the three FPs. When comparing each single fore-period between both groups, post-hoc comparisons showed that RTs decreased significantly after mental training. Significant differences were found for shortest FP (650 ms) between "woMT" and "wMT" mean = $377.49, \mathrm{SD}=147.91,95 \% \mathrm{CI}=[362.47 ; 392.50], p=0.001$. No difference was found for the longest FP (680 ms and 710 $\mathrm{ms})$.

\section{Imaging results}

In the contrast "wMT" > "woMT" (two-sample $t$-test), activations were significantly enhanced for both alertness conditions. Within exogenous alertness condition, activations were mainly increased in the right hemisphere: anterior insula (AI), anterior cingulate cortex (ACC), inferior parietal cortex (IPC), thalamus, dorsolateral prefrontal cortex (DLPFC), and Putamen. Within endogenous alertness condition: similar activations were observed in addition to bilateral activations in IPC and putamen (Table 1; Figure 3).

\section{Effective connectivity results}

During exogenous alertness condition in the "wMT" group, results showed significantly increased effective connectivity between AI (x: 39, y: 2, z: 10) and DLPFC (x: 51, y: 29, z: 31) $p F W E=0.001$ and between $\operatorname{AI}(39, \mathrm{y}: 2, \mathrm{z}: 10)$ and ACC (x: 9, $\mathrm{y}: 41, \mathrm{z}: 7) p F W E=0.01$ for the shortest FP $(250 \mathrm{~ms})$. No 

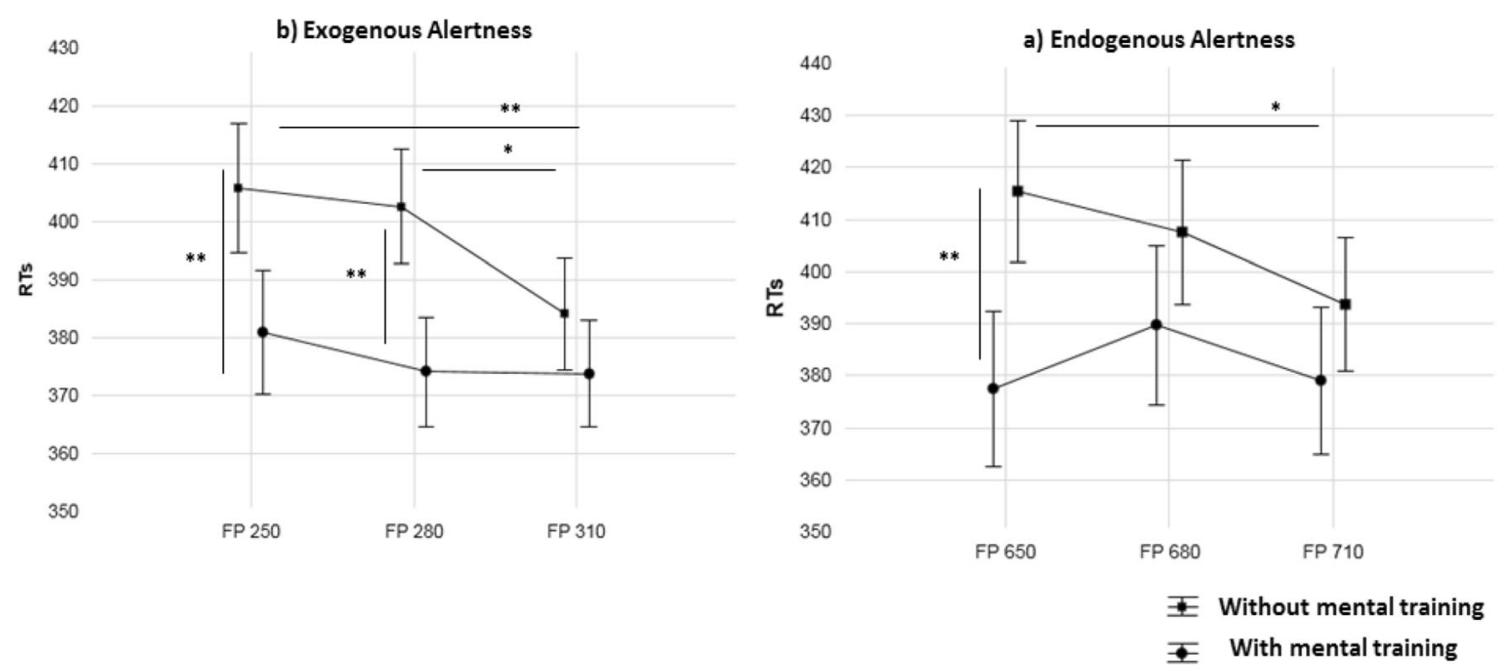

Fig. 2 Results of ANOVA [Groups x Fore-periods] during exogenous (a) and endogenous (b) alertness tasks. $* * p<0.01 ; * p<0.05$

increased effective connectivity was found for the longer FP (310 ms). During endogenous alertness condition, in the "wMT" group, results showed significantly increased effective connectivity between DLPFC (x: 57, y: 32, z: 19) and ACC (x: 3, y: 41, z: 7) $p F W E=0.005$ for shortest FP (650 ms) (Figures 4 and 5). No significant increased effective connectivity was found for longer FP (710 ms).

Table 1 Results of two-sample $t$-test of activated regions for the exogenous and endogenous alerting contrast with mental training $>$ without mental training; Regions side (R/L), Montreal Neurological Institute (MNI) coordinates, FWE-corrected $(p<0.05)$

\begin{tabular}{|c|c|c|c|c|c|c|c|}
\hline Regions & Side & $B A$ & $\mathrm{x}$ & $\mathrm{y}$ & $\mathrm{z}$ & $\mathrm{t}$ & $\begin{array}{l}\mathrm{P}(\mathrm{FWE}- \\
\text { corr) }\end{array}$ \\
\hline \multicolumn{8}{|c|}{ Exogenous alertness } \\
\hline$I P C$ & $\mathbf{R}$ & BA 40 & 42 & -46 & 46 & 4.5 & 0.007 \\
\hline$A C C$ & $\mathbf{R}$ & BA 32 & 6 & 26 & -8 & 4.43 & 0.009 \\
\hline$D L P F C$ & $\mathbf{R}$ & BA 46 & 45 & 26 & 22 & 4.92 & 0.004 \\
\hline insula & $\mathbf{R}$ & BA13 & 51 & -43 & 19 & 5.61 & 0.001 \\
\hline Thalamus & $\mathbf{R}$ & & 15 & -10 & 10 & 4.27 & 0.01 \\
\hline Putamen & $\mathbf{R}$ & & 27 & 2 & 7 & 3.88 & 0.03 \\
\hline \multicolumn{8}{|c|}{ Endogenous alertness } \\
\hline$A C C$ & & & 6 & 44 & -2 & 6.01 & 0.0004 \\
\hline$I P C$ & $\mathbf{R}$ & BA 40 & 48 & -37 & 37 & 3.97 & 0.01 \\
\hline- & $\mathbf{L}$ & BA 40 & -57 & -28 & 31 & 3.69 & 0.02 \\
\hline$D L P F C$ & $\mathbf{R}$ & BA 46 & 42 & 35 & 19 & 4.82 & 0.001 \\
\hline insula & $\mathbf{R}$ & BA 13 & 42 & 41 & 1 & 5.07 & 0.006 \\
\hline thalamus & $\mathbf{L}$ & & -3 & -7 & 1 & 3.9 & 0.03 \\
\hline Putamen & $\mathbf{L}$ & & -21 & 11 & -5 & 5.29 & 0.001 \\
\hline- & $\mathbf{R}$ & & 30 & -16 & 4 & 4.96 & 0.003 \\
\hline
\end{tabular}

\section{Discussion}

This study provides evidence that a temporal deployment of attention by mental training, particularly optimized in short time scales, reinforces dynamically both top-down (endogenous alertness) and bottom-up (exogenous alertness) networks responsible for the control of attention.

Our behavioral results provide clear evidence of FPs effect in exogenous and endogenous alertness. Within the "woMT" group, RTs are significantly shorter for long FPs. Indeed, during endogenous condition, a warning stimulus is involved. This one informs the participant of the upcoming stimulus. A real delay of preparation is omnipresent in the task. The longer the interval between the temporal cue (black screen) and the imperative stimulus (white square) is, the better is the temporal preparation, especially for longer FP $(710 \mathrm{~ms})$. This result goes in line with many studies in which the warning stimulus is known to increase probability to respond at the prominent moment (Perin et al., 2010). Likewise, for exogenous alertness condition, RTs were shorter for longer FP $(310 \mathrm{~ms})$ relatively to $(250 \mathrm{~ms})$ and $(280 \mathrm{~ms})$. This result was not necessarily expected because exogenous alertness does not engage a real process of preparation. This result goes with some other studies (Hackley et al., 2009; Kiesel, Miller, \& Ulrich, 2007; Stahl \& Rammsayer, 2005) in which accessory stimulus (in our case the cross presented with randomized FPs) still produces a benefit on RTs even though there is no explicit temporal preparation in this situation. This FP effect disappeared within the "wMT" group, and no significant difference of RT was observed between the three FPs within both alertness conditions. We demonstrate that the 

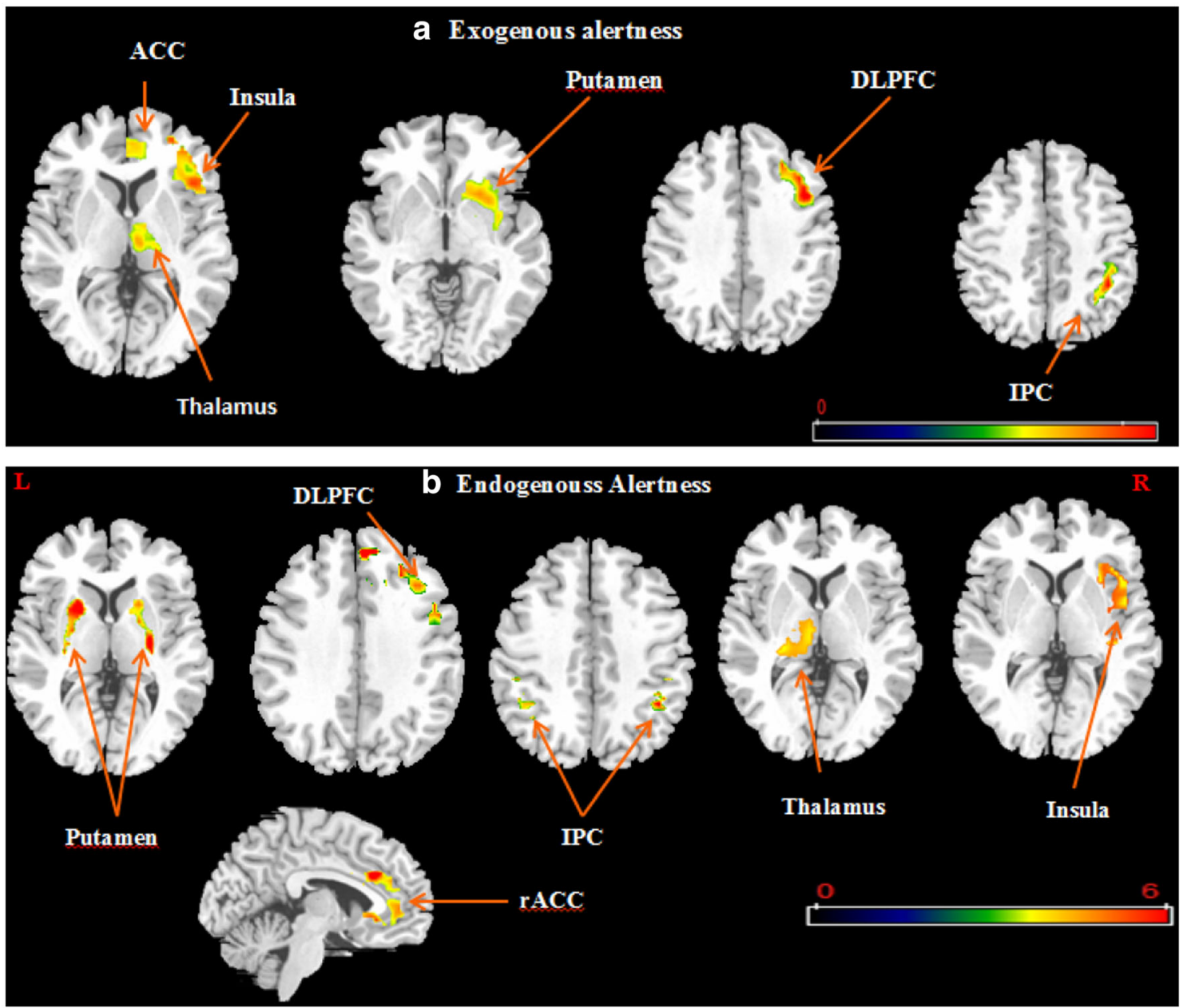

Fig. 3 (A) Brain areas maps, associated with "wMT> woMT" contrast, showing significant activations within exogenous alertness in the right hemisphere (ACC, DLPFC, insula, thalamus, and IPC. (B) In endogenous task, significant activations were observed in the right
DLPFC, ACC and insula, bilaterally in the putamen and IPC and finally in the left thalamus. The resulting activations were thresholded at FWE-corrected $p$ values $(p<0.05)$

the trials, the higher the conditioned strength at a critical moment, the shorter the RT will be if the imperative stimulus appears at that moment. It would be reasonable to assume that mental rehearsal has increased the "conditioned strength" during trials increasing consequently the predictability of target occurrence only for short FPs conversely to longer ones in which temporal readiness has remained unchanged and no RT improvement has been seen. This assumption goes also with the theoretical conception of Los and Van Den Heuvel (2001) who consider that after some acquisition training, a conditioned response is developed and locked in time andobtains its peak at or about the moment the unconditioned stimulus is presented (Gallistel \& Gibbon, 2000; Grossberg \& Merrill, 1992; Roberts, 1998).

We also may interpret our findings as a function of arousal modulation, because the state of preparation is in part determined by the level of arousal. Arousal and conditioned strength are two dependent components (Vallesi, Shallice, \& Walsh, 2007). According to Vallesi et al. (2007), the higher 


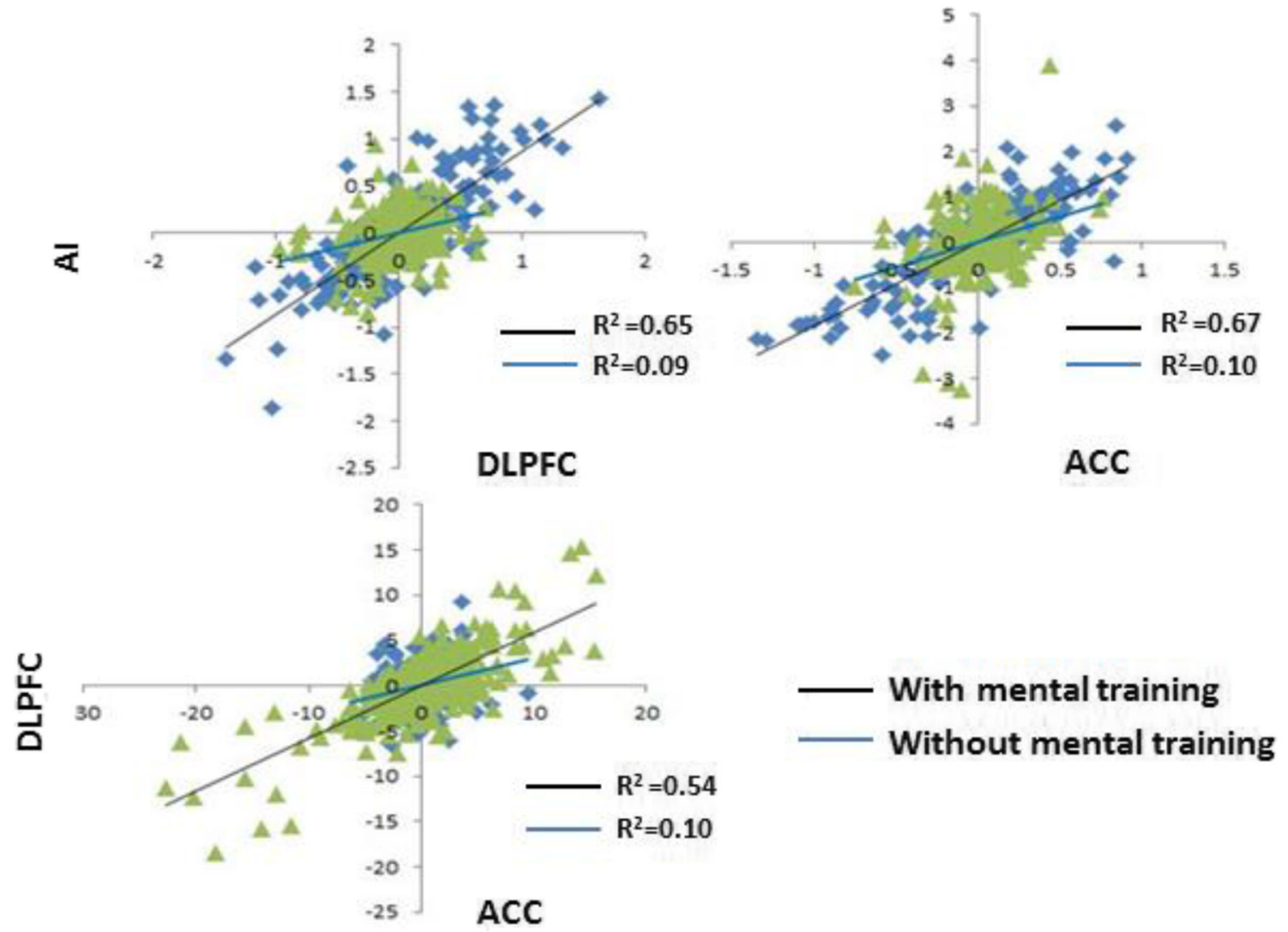

Fig. 4 Results of the psycho-physiological interactions. Slopes found between AI-ACC, AI-DLPFC, and DLPFC-ACC in the context "with/ without mental training" are significantly different $(p<0.05$, FWEcorrected). PPI show, within "wMT" group, significant increased interaction in the right hemisphere between $\mathrm{AI}$ and DLPFC and between $\mathrm{AI}$ and ACC during exogenous alertness condition for $250 \mathrm{~ms}$ FP. During endogenous alertness condition, effective connectivity was significantly increased between DLPFC and ACC for the shortest FP (650ms) the conditioned strength, the greater the level of participant's arousal would be. These authors assume that arousal tends to increase particularly during short FPs than long ones. Our findings are in line with this conceptualization. With mental training, for longer FPs, we may assume that arousal has already reached its maximal peak and could not exceed this optimal level. We also suggest that the session of mental imagery (motor inhibition) activates the autonomic nervous system (ANS), which is permanently and intensively stimulated, increasing the arousal level and facilitating the encoding of information (notably via the insula). Over time, the probability that the targets appears imminently increases and consequently its uncertainty diminishes. During the physical (real) task performed a few minutes after mental training, we hypothesize that cognitivo-motor loops or mnesic traces are quickly exacerbated (reactivated) thanks to attentional (rehearsal) learning, which reinforces the motor and cognitive 1 controls and improves the behavioral performance (decreasing RT during the shortest FPs).

Our imaging data show that DLPFC, ACC, and AI activity was broadly increased with mental training. In addition, PPI analysis highlights three neural circuits that were enhanced with mental training: tDLPFC-ACC (during endogenous condition) and AI-ACC/AI-DLPFC (during exogenous condition). On top of that, neuropsychological tests of attention show that performance has become better within the "wMT" group, which means that processing speed capacities have become better after mental training.

Our study provides evidence that temporal processing of alertness, whether exogenous or endogenous, is generated by a common neural basis following a coupled or crosscontrol "top down and bottom up," which depends on the nature of the task. Actually, in the brain, the control of exogenous alertness is related to autonomic nervous system or arousal mediated by noradrenergic and cholinergic systems originating from the ponto-mesencephalic part of the brainstem (Clemens et al., 2011; Gaspar, Berger, Febvret, Vigny, \& Henry, 1989; Jones, 2003; Robinson \& Coyle, 1980). So, exogenous alertness involves uncontrolled automatic mechanisms rather than voluntary control processes. It is considered an adaptive system that includes intuition and unconscious mechanisms, which evolve according to homeostatic changes. With attentional induction, these mechanisms seem to be adjusted much faster. This automatic adjustment appears to be more relevant for shorter FP in which the 


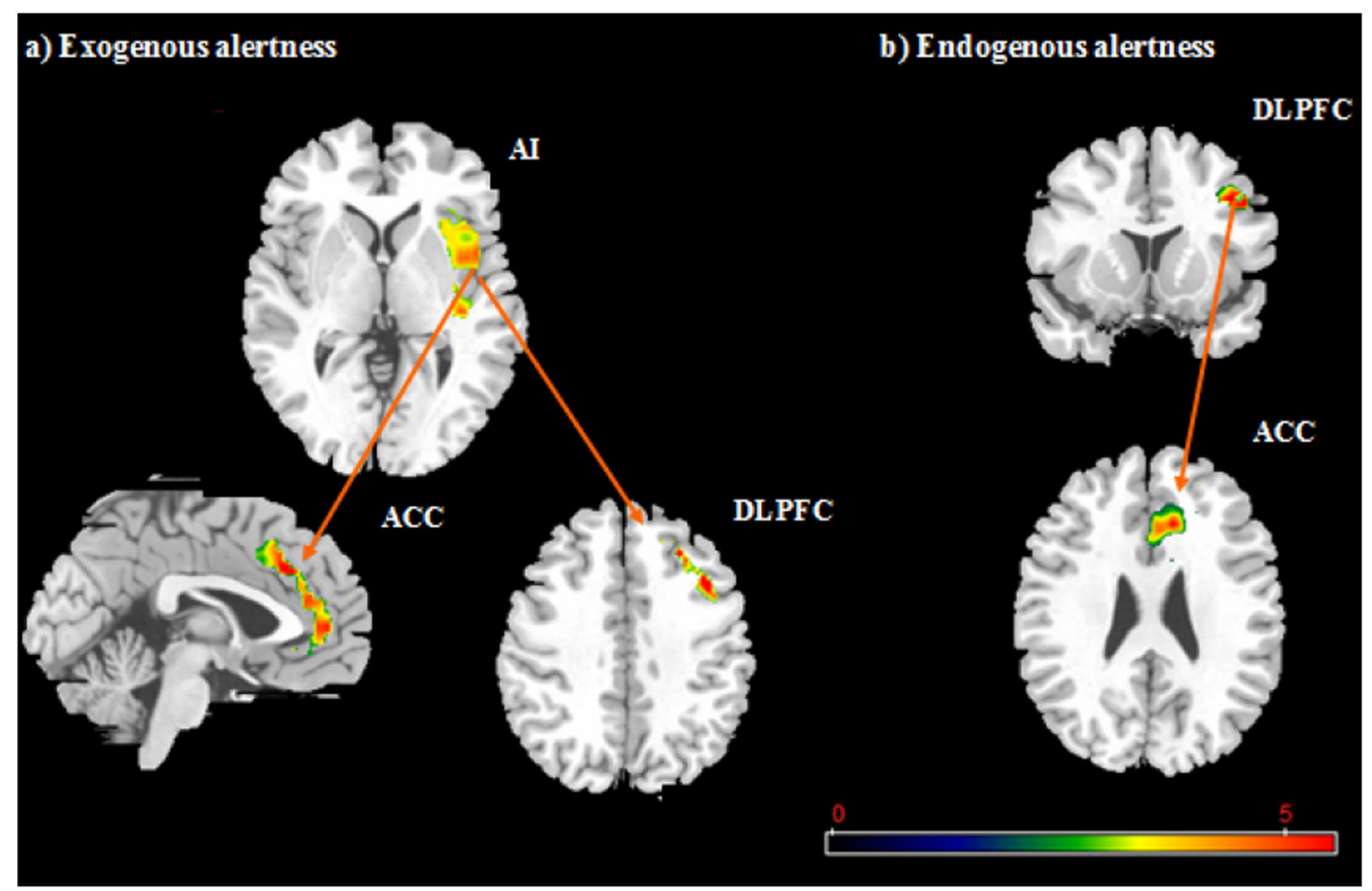

Fig. 5 Brain areas maps associated with psycho-physiological interactions. PPI results show for "wMT" group significant increased interaction in the right hemisphere between AI and DLPFC and between AI and ACC for the shortest during exogenous alertness condition for FP (250 ms). During endogenous alertness condition, effective connectivity was significantly increased between DLPFC and ACC for the shortest FP (650 $\mathrm{ms})$ miniature vigilance bursts briefly and improves significantly the performance (Posner and Bois 1971). Furthermore, AI known to play a higher net causal outflow, helps to generate a bottom-up control involved in the transfer of fast control signals, and stable maintenance of task mode and strategy (Cauda et al., 2011; Menon \& Uddin, 2010). Despite the very short lapse of time $(<300 \mathrm{~ms})$ between the fixation cross (accessory stimulus) and the imperative stimulus (square), anterior insula may facilitate information temporal processing under attentional induction and this by providing relevant control signals to engage brain regions responsible for attention adjustment (ACC and DLPFC). This result is of great interest, because it may constitute a theoretical basis for further studies on clinical populations for example, such as multiple sclerosis which suffers the inability to sustain and orient attention to a given point, exhaustion, and fatigue, which seem more related to automatic physiological processes rather than higher-order mechanisms.

Endogenous alertness is related to high cognitive control (discrimination and selection processing during the temporal preparation). During this condition, the DLPFC is known to play a fundamental role in the organization and preparation of forthcoming actions. Based on information stored in the working memory (Pochon, 2001), the DLPFC exerts a top-down control on ACC when competition between responses increases and when inhibition of motor response is required (Dreher \& Grafman, 2003). The DLPFC is responsible for maintaining a high-level of preparation during waiting interval, whereas the ACC appears to be more directly involved in inhibition of responses, control monitoring, and conflict resolution (Garavan, Ross, Murphy, Roche, \& Stein, 2002). Via this top-down control, ACC may inhibit responses during delay period "the proactive inhibition" and prepares for the real motor execution at the right moment "the proponent response" (Narayanan \& Laubach, 2009; Picard \& Strick, 2001). This process may be related to two pools of antagonist neurons (excitatory and inhibitory), which interact continuously and synchronously during trials.

Furthermore, our finding proposes a putative neural model including three hub regions (AI, ACC, and DLPFC) known to share a particular population of neurons: the Von Economo Neurons (VENs). The average population of VENs in the $\mathrm{ACC}$ and $\mathrm{AI}$ is about five times larger than the average layer 5 pyramidal cells (Gaspar et al., 1989) and axon calibers of the VENs are larger and faster conducting than in the neighboring pyramids since conduction speed is proportional to axonal diameter. The large size and simple dendritic architecture of the VENs supports the conjecture that they are built for speed. 
Initially, these neurons were related to social cognition, awareness, and intuition in which many variables are rapidly evaluated to yield a fast decision (Allman et al., 2010; Allman, Watson, Tetreault, \& Hakeem, 2005; Craig, 2009). They have been suggested to rapidly adjust behavior in quickly changing situations, and this puts a premium on the capacity to respond quickly to changing conditions and facilitates the rapid execution of decisions (Allman et al., 2005). Based on the fundamental characteristics of the VENs (speed processing and rapid conduction), we speculate with caution that VENs may constitute a neural basis of fast control signals not only for social changing situation but also for quick temporal pressure when faced with risky decision, uncertainty or thereat.

We do not have any empirical evidence about the implication of these neurons in attentional tasks. We have postulated this hypothesis relying on the roles that were attributed to these neurones and their anatomical architecture. We suggest that, densely present in the AI, ACC, and DLPFC, they may not be restricted to the social domain and may intervene in speed and temporal processing of the attentional control. More explicitly, we think that these neurons could potentially constitute a neural mechanism of action, and with attentional induction coming from mental training may increase firing particularly during the short delay periods (FPs) decreasing uncertainty and facilitate decision making in time. Others investigations (at a microscopic level) should be realized to consolidate this neural hypothesis, because fMRI as an investigative technique does not support this type of hypothesis at a macroscopic level.

Finally, it is known from literature that there are gender differences on cognitive control during attentional tasks. The attentional effects differ according to the nature of the cognitive task and its difficulties (Der \& Deary, 2006; Riley et al., 2016). In our study, using the gender as a categorical controlling variable, a significant effect of gender has been observed in the global analysis of 40 subjects without separating FPs. Results show significant interaction (Alertness $\mathrm{x}$ Gender) $F(1,23)=20.693, p<10^{-5}$ with very low size effect: partial $\eta^{2}=0.009$. Post-hoc comparisons showed that RTs were shorter within females $(F)$ compared with males $(\mathrm{M})$ in both alertness conditions: endogenous condition (mean $\mathrm{F}=339.57$; $\mathrm{SD}=106.02$; mean $\mathrm{M}=394.41 ; \mathrm{SD}=$ 155.52 ), $p<10^{-5}$; exogenous condition (mean $\mathrm{F}=375.03 ; \mathrm{SD}=$ 92.50; mean $\mathrm{M}=403.66$; $\mathrm{SD}=122.46, p<10^{-5}$. Also, a significant interaction was observed between (Groups x Gender) $F(1$, 50) $=20.63, p=0.00001$ with very low size effect: partial $\eta^{2}=$ 0.006 . Post hoc comparisons showed that RT were shorter within wMT group particularly for females (mean woMT $=381.73$; SD = 95.86; mean wMT = 332.96; $\mathrm{SD}=99.84), p<10^{-5}$. No significant differences were found for males. Similar statistical analyzes have been conducted within (Groups x FPs) within each alertness condition, including gender as a covariable. Results show no significant gender effect on separate FPs, probably because of the small size of the sample $(\mathrm{n}=10$ females, $\mathrm{n}=10$ males). It would be of interest in a future study to investigate gender differences using a larger sample size to show gender effects on the attentional control and in particular on the temporal processing of the information which would strengthen our neural hypotheses.

\section{Conclusions and perspectives}

The present finding confirms the studies on classic FP effect and provides surprising and innovative neuropsychological insight on the temporal deployment of attention, suggesting that mentaltraining of alertness using short training sessions could ameliorate the behavioral performance and reinforce the concentration. These findings strongly support the utility of using such protocol within clinical populations, such patients with chronic fatigue, stroke, multiple sclerosis (MS), and amyotrophic lateral sclerosis (ALS), known to experience difficulties in sustaining attention (vigilance) and temporally prepare for forthcoming actions, but also to disentangle the effects of mental and physical fatigue. Patients with MS often report that fatigue adversely affects their cognitive functioning. MS patients are known to suffer from attentional impairments, such the inability to sustain attention for a long time and experience difficulties in complex divided attentional controls and orient attention to a given point (De Luca 2008). Also, impairments of information processing, particularly deficits in information processing speed have been reported (Rao, Leo, Bernardin, \& Unverzagt, 1991; Snyder, Cappelleri, Archibald, \& Fisk, 2001). The most important finding of our study is that we demonstrated that a short and intensive session of mental training increases attentional resources (arousal) and induces a temporal deployment of attention by increasing information processing speed. We also showed that mental training has strengthened connectivity between neural circuits responsible basically for the control of attention during short FPs intervals. In this context, we suggest that attentional mental training during a short and intensive session could be a useful rehabilitation method within MS population to reinforce the attentional control and the information cognitive processing. Also, the neural circuits investigated in this study could play the role of biomarker of central and peripheral fatigue in this population. This finding could be not restricted to MS patients but also to other clinical populations, such Amyotrophic Lateral Sclerosis (ALS) patients who suffer from planification, initiation and execution of movement (Abidi et al., 2020; Feron et al., 2018). Visual (auditive) motor imagery with attentional and motor control used as mental training task would reinforce altered sensorimotor and cognitive circuits in ALS patients, thereby improving initiation and execution of motion in this population. 
Acknowledgements This work was partly supported and by LINP 2 laboratory, Université Paris Nanterre, F92000 Nanterre, France and the COMUE University Paris Lumière (UPL). The authors thank Dr. Marie de Montalembert who provided the neuropsychological batteries. This research did not receive any specific grant from funding agencies in the public, commercial, or not-for-profit sectors. Our best thanks to General Electric, Buc France for the partnership sealed with Pontoise Hospital Imaging Centre (Pr. A. F. Le Blanche) in complimentary providing BrainWave RT software. None of the data or materials for the experiments reported are available, and none of the experiments were preregistered.

\section{Compliance with ethical standards}

Conflict of interest The authors of this manuscript declare that they have no conflict of interest to disclose.

\section{References}

Abidi, M., de Marco, G., Couillandre, A., Feron, M., Mseddi, E., Termoz, N., ... Bede, P. (2020). Adaptive functional reorganization in amyotrophic lateral sclerosis: coexisting degenerative and compensatory changes. European Journal of Neurology https://doi.org/10.1111/ ene. 14042

Allman, J. M., Tetreault, N. A., Hakeem, A. Y., Manaye, K. F., Semendeferi, K., Erwin, J. M., ... Hof, P. R. (2010). The von Economo neurons in frontoinsular and anterior cingulate cortex in great apes and humans. Brain Structure \& Function https://doi.org/ 10.1007/s00429-010-0254-0

Allman, J. M., Watson, K. K., Tetreault, N. A., \& Hakeem, A. Y. (2005). Intuition and autism: A possible role for Von Economo neurons. Trends in Cognitive Sciences, 9(8), 367-373. https://doi.org/10. 1016/j.tics.2005.06.008

Barbot, A., Landy, M. S., \& Carrasco, M. (2012). Differential effects of exogenous and endogenous attention on second-order texture contrast sensitivity. Journal of Vision. https://doi.org/10.1167/12.8.6

Bates, M. E., \& Lemay, E. P. (2004). The d2 test of attention: Construct validity and extensions in scoring techniques. Journal of the International Neuropsychological Society. https://doi.org/10.1017/ S135561770410307X

Bowie, C. R., \& Harvey, P. D. (2006). Administration and interpretation of the Trail Making Test. Nature Protocols. https://doi.org/10.1038/ nprot. 2006.390

Brunia, C. H. M., De Jong, B. M., Van Den Berg-Lenssen, M. M. C., \& Paans, A. M. J. (2000). Visual feedback about time estimation is related to a right hemisphere activation measured by PET. Experimental Brain Research, 130(3), 328-337. https://doi.org/10. $1007 / \mathrm{s} 002219900293$

Capizzi, M., Sanabria, D., \& Correa, Á. (2012). Dissociating controlled from automatic processing in temporal preparation. Cognition, 123(2), 293-302. https://doi.org/10.1016/j.cognition.2012.02.005

Carrasco, M. (2011). Visual attention: The past 25 years. Vision Research. https://doi.org/10.1016/j.visres.2011.04.012

Cauda, F., D'Agata, F., Sacco, K., Duca, S., Geminiani, G., \& Vercelli, A. (2011). Functional connectivity of the insula in the resting brain. NeuroImage, 55(1), 8-23. https://doi.org/10.1016/j.neuroimage. 2010.11.049

Clemens, B., Zvyagintsev, M., Sack, A., Heinecke, A., Willmes, K., \& Sturm, W. (2011). Revealing the functional neuroanatomy of intrinsic alertness using fMRI: Methodological peculiarities. PLOS ONE, 6(9). https://doi.org/10.1371/journal.pone.0025453

Clemens, B., Zvyagintsev, M., Sack, A. T., Heinecke, A., Willmes, K., \& Sturm, W. (2013). Comparison of fMRI activation patterns for test and training procedures of alertness and focused attention. Restorative Neurology and Neuroscience, 31(3), 311-336. https:// doi.org/10.3233/RNN-120266

Cohen, J. (2013). Statistical Power Analysis for the Behavioral Sciences. In Statistical Power Analysis for the Behavioral Sciences. https:// doi.org/10.4324/9780203771587

Corrigan, J. D., \& Hinkeldey, N. S. (1987). Relationships between Parts A and B of the Trail Making Test. Journal of Clinical Psychology. https://doi.org/10.1002/1097-4679(198707)43:4<402::AIDJCLP2270430411>3.0.CO;2-E

Coste, C. P., \& Kleinschmidt, A. (2016). Cingulo-opercular network activity maintains alertness. NeuroImage. https://doi.org/10.1016/j. neuroimage.2016.01.026

Craig, A. D. (2002). Opinion: How do you feel? Interoception: the sense of the physiological condition of the body. Nature Reviews.

Craig, A. D., (2005). Forebrain emotional asymmetry: A neuroanatomical basis? Trends in Cognitive Sciences. https://doi.org/10.1016/j.tics. 2005.10.005

Craig, A. D. (2009). Emotional moments across time: A possible neural basis for time perception in the anterior insula. Philosophical Transactions of the Royal Society B: Biological Sciences, 364(1525), 1933-1942. https://doi.org/10.1098/rstb.2009.0008

Critchley, H. D., Melmed, R. N., Featherstone, E., Mathias, C. J., \& Dolan, R. J. (2002). Volitional control of autonomic arousal: A functional magnetic resonance study. NeuroImage. https://doi.org/ 10.1006/nimg.2002.1147

Decety, J., \& Grèzes, J. (1999). Neural mechanisms subserving the perception of human actions. Trends in Cognitive Sciences. https://doi. org/10.1016/S1364-6613(99)01312-1

DeLuca, J., Genova, H. M., Hillary, F. G., \& Wylie, G. (2008). Neural correlates of cognitive fatigue in multiple sclerosis using functional MRI. Journal of the Neurological Sciences. https://doi.org/10.1016/ j.jns.2008.01.018

Denney, D. R., \& Lynch, S. G. (2009). The impact of multiple sclerosis on patients' performance on the Stroop Test: Processing speed versus interference. Journal of the International Neuropsychological Society. https://doi.org/10.1017/S1355617709090730

Der, G., \& Deary, I. J. (2006). Age and sex differences in reaction time in adulthood: Results from the United Kingdom health and lifestyle survey. Psychology and Aging. https://doi.org/10.1037/0882-7974. 21.1 .62

Dreher, J. C., \& Grafman, J. (2003). Dissociating the roles of the rostral anterior cingulate and the lateral prefrontal cortices in performing two tasks simultaneously or successively. Cerebral Cortex. https:// doi.org/10.1093/cercor/13.4.329

Dye, M. W. G., Green, C. S., \& Bavelier, D. (2009). The development of attention skills in action video game players. Neuropsychologia. https://doi.org/10.1016/j.neuropsychologia.2009.02.002

Feron, M., Couillandre, A., Mseddi, E., Termoz, N., Abidi, M., Bardinet, E., ... de Marco, G. (2018). Extrapyramidal deficits in ALS: a combined biomechanical and neuroimaging study. Journal of Neurology. https://doi.org/10.1007/s00415-018-8964-y

Friston, K. J., Buechel, C., Fink, G. R., Morris, J., Rolls, E., \& Dolan, R. J. (1997). Psychophysiological and modulatory interactions in neuroimaging. NeuroImage. https://doi.org/10.1006/nimg.1997.0291

Gallistel, C. R., \& Gibbon, J. (2000). Time, rate, and conditioning. Psychological Review. https://doi.org/10.1037/0033-295X.107.2. 289

Garavan, H., Ross, T. J., Murphy, K., Roche, R. A. P., \& Stein, E. A. (2002). Dissociable executive functions in the dynamic control of behavior: Inhibition, error detection, and correction. NeuroImage. https://doi.org/10.1006/nimg.2002.1326

Gaspar, P., Berger, B., Febvret, A., Vigny, A., \& Henry, J. P. (1989). Catecholamine innervation of the human cerebral cortex as revealed by comparative immunohistochemistry of tyrosine hydroxylase and 
dopamine-beta-hydroxylase. Journal of Comparative Neurology. https://doi.org/10.1002/cne.902790208

Gaudino, E. A., Geisler, M. W., \& Squires, N. K. (1995). Construct Validity in the Trail Making Test: What Makes Part B Harder? Journal of Clinical and Experimental Neuropsychology. https:// doi.org/10.1080/01688639508405143

Golden, C. J. (1976). Identification of brain disorders by the Stroop Color and Word Test. Journal of Clinical Psychology.

Grossberg, S., \& Merrill, J. W. L. (1992). A neural network model of adaptively timed reinforcement learning and hippocampal dynamics. Cognitive Brain Research. https://doi.org/10.1016/09266410(92)90003-A

Guillot, A., Collet, C., Nguyen, V. A., Malouin, F., Richards, C., \& Doyon, J. (2009). Brain activity during visual versus kinesthetic imagery: An fMRI study. Human Brain Mapping. https://doi.org/ 10.1002/hbm. 20658

Hackley, S. A., Langner, R., Rolke, B., Erb, M., Grodd, W., \& Ulrich, R. (2009). Separation of phasic arousal and expectancy effects in a speeded reaction time task via fMRI. Psychophysiology, 46(1), 163-171. https://doi.org/10.1111/j.1469-8986.2008.00722.x

Han, S. W., Shin, H., Jeong, D., Jung, S., Bae, E., Kim, J. Y., ... Kim, K. (2018). Neural substrates of purely endogenous, self-regulatory control of attention. Scientific Reports, 8(1), 1-10. https://doi.org/10. 1038/s41598-018-19508-6

Hsieh, Y. H., Chen, K. J., Wang, C. C., \& Lai, C. L. (2008). Cognitive and motor components of response speed in the Stroop test in Parkinson's disease patients. Kaohsiung Journal of Medical Sciences. https://doi.org/10.1016/S1607-551X(08)70117-7

Jackson, P. L., Lafleur, M. F., Malouin, F., Richards, C., \& Doyon, J. (2001). Potential role of mental practice using motor imagery in neurologic rehabilitation. Archives of Physical Medicine and Rehabilitation. https://doi.org/10.1053/apmr.2001.24286

Johnson, J. G., \& Raab, M. (2003). Take the first: Option-generation and resulting choices. Organizational Behavior and Human Decision Processes. https://doi.org/10.1016/S0749-5978(03)00027-X

Jones, B. E. (2003). [Frontiers in Bioscience 8, s438-451, May 1, 2003] AROUSAL SYSTEMS Barbara E. Jones. Social Biology. https:// doi.org/10.2741/1074

Kahneman, D., \& Klein, G. (2009). Conditions for Intuitive Expertise: A Failure to Disagree. American Psychologist. https://doi.org/10.1037/ a0016755

Kiesel, A., Miller, J., \& Ulrich, R. (2007). Systematic biases and Type i error accumulation in tests of the race model inequality. Behavior Research Methods. https://doi.org/10.3758/BF03193024

Klein, G., Calderwood, R., \& Clinton-Cirocco, A. (2010). Rapid Decision Making on the Fire Ground: The Original Study Plus a Postscript. Journal of Cognitive Engineering and Decision Making. https://doi.org/10.1518/155534310x12844000801203

Los, S. A., Kruijne, W., \& Meeter, M. (2014). Outlines of a multiple trace theory of temporal preparation. Frontiers in Psychology, 5(SEP), 113. https://doi.org/10.3389/fpsyg.2014.01058

Los, S. A., \& Van Den Heuvel, C. E. (2001). Intentional and unintentional contributions to nonspecific preparation during reaction time foreperiods. Journal of Experimental Psychology: Human Perception and Performance. https://doi.org/10.1037/0096-1523. 27.2.370

Menon, V., \& Uddin, L. Q. (2010). Saliency, switching, attention and control: a network model of insula function. Brain Structure \& Function. https://doi.org/10.1007/s00429-010-0262-0

Mottaghy, F. M., Willmes, K., Horwitz, B., Müller, H. W., Krause, B. J., \& Sturm, W. (2006). Systems level modeling of a neuronal network subserving intrinsic alertness. NeuroImage. https://doi.org/10.1016/ j.neuroimage.2005.07.034

Muller, K., \& Cohen, J. (1989). Statistical Power Analysis for the Behavioral Sciences. Technometrics. https://doi.org/10.2307/ 1270020
Narayanan, N. S., \& Laubach, M. (2009). Delay Activity in Rodent Frontal Cortex During a Simple Reaction Time Task. Journal of Neurophysiology, 101(6), 2859-2871. https://doi.org/10.1152/jn. 90615.2008

Niemi, P., \& Näätänen, R. (1981). Foreperiod and simple reaction time. Psychological Bulletin. https://doi.org/10.1037/0033-2909.89.1.133

Nobre, A. C., \& Coull, J. T. (2010). How can temporal expectations bias perception and action? In Attention and Time. https://doi.org/10. 1093/acprof:oso/9780199563456.003.0027

Olivier, I., \& Rival, C. (2002). Foreperiod duration and motor preparation during childhood. Neuroscience Letters. https://doi.org/10.1016/ S0304-3940(01)02510-1

Pena-Casanova, J., Blesa, R., Aguilar, M., Gramunt-Fombuena, N., Gomez-Anson, B., Oliva, R., ... Sol, J. M. (2009). Spanish Multicenter Normative Studies (NEURONORMA Project): Methods and Sample Characteristics. Archives of Clinical Neuropsychology. https://doi.org/10.1093/arclin/acp027

Périn, B., Godefroy, O., Fall, S., \& de Marco, G. (2010). Alertness in young healthy subjects: An fMRI study of brain region interactivity enhanced by a warning signal. Brain and Cognition, 72(2), 271281. https://doi.org/10.1016/j.bandc.2009.09.010

Picard, N., \& Strick, P. L. (2001). Imaging the premotor areas. Current Opinion in Neurobiology. https://doi.org/10.1016/S0959-4388(01) 00266-5

Pochon, J.-B. (2001). The Role of Dorsolateral Prefrontal Cortex in the Preparation of Forthcoming Actions: an fMRI Study. Cerebral Cortex, 11(3), 260-266. https://doi.org/10.1093/cercor/11.3.260

Posner, Michael I, \& Rafal, R. D. (1987). Cognitive theories of attention and the rehabilitation of attentional deficits. In Neuropsychological Rehabilitation 182-201

Posner, M. I., Cohen, Y., \& Rafal, R. D. (1982). Neural systems control of spatial orienting. Philosophical Transactions of the Royal Society of London. Series B, Biological Sciences. https://oi.org/10.1098/ rstb.1982.0081

Posner, M. I. and S. J. B. (1971). Components of Attention. Psychological Review, 78(5), 391-408. https://doi.org/10.1037/ h0031333

Rao, S. M., Leo, G. J., Bernardin, L., \& Unverzagt, F. (1991). Cognitive dysfunction in multiple sclerosis. I. Frequency, patterns, and prediction. Neurology. https://doi.org/10.1212/WNL.41.5.685

Riley, E., Okabe, H., Germine, L., Wilmer, J., Esterman, M., \& Degutis, J. (2016). Gender differences in sustained attentional control relate to gender inequality across countries. PLoS ONE. https://doi.org/10. 1371/journal.pone. 0165100

Roberts, S. (1998). The mental representation of time: uncovering a biological clock.pdf. In An Invitation to Cognitive Science - methods, models, and conceptual issues.

Robinson, R. G., \& Coyle, J. T. (1980). The differential effect of right versus left hemispheric cerebral infarction on catecholamines and behavior in the rat. Brain Research. https://doi.org/10.1016/00068993(80)90557-0

Rogers, L. J., \& Andrew, R. J. (2002). The Nature of Lateralisation in Tetrapods. In Comparative Vertebrate Lateralisation. https://doi.org/ 10.1017/CBO9780511546372

Sadaghiani, S., \& D'Esposito, M. (2015). Functional characterization of the cingulo-opercular network in the maintenance of tonic alertness. Cerebral Cortex, 25(9), 2763-2773. https://doi.org/10.1093/cercor/ bhu072

Snyder, P. J., Cappelleri, J. C., Archibald, C. J., \& Fisk, J. D. (2001). Improved detection of differential information-processing speed deficits between two disease-course types of multiple sclerosis. Neuropsychology. https://doi.org/10.1037/0894-4105.15.4.617

Sridharan, D., Levitin, D. J., \& Menon, V. (2008). A critical role for the right fronto-insular cortex in switching between central-executive and default-mode networks. Proceedings of the National Academy of Sciences. https://doi.org/10.1073/pnas.0800005105 
Stahl, J., \& Rammsayer, T. H. (2005). Accessory stimulation in the time course of visuomotor information processing: Stimulus intensity effects on reaction time and response force. Acta Psychologica. https://doi.org/10.1016/j.actpsy.2005.02.003

Steinborn, M. B., Langner, R., Flehmig, H. C., \& Huestegge, L. (2018). Methodology of performance scoring in the $\mathrm{d} 2$ sustained-attention test: Cumulative-reliability functions and practical guidelines. Psychological Assessment. https://doi.org/10.1037/pas0000482

Sturm, W., De Simone, A., Krause, B. J., Specht, K., Hesselmann, V., Radermacher, I., ... Willmes, K. (1999). Functional anatomy of intrinsic alertness: Evidence for a fronto-parietal-thalamicbrainstem network in the right hemisphere. Neuropsychologia, 37(7), 797-805. https://doi.org/10.1016/S0028-3932(98)00141-9

Sturm, W, Willmes, K. (2001). On the functional neuroanatomy of intrinsic and phasic alertness. NeuroImage, 14(1 II), 76-84. https:// doi.org/10.1006/nimg.2001.0839

Tombaugh, T. N. (2004). Trail Making Test A and B: Normative data stratified by age and education. Archives of Clinical Neuropsychology. https://doi.org/10.1016/S0887-6177(03)00039-8

Vallesi, A., \& Shallice, T. (2007). Developmental Dissociations of Preparation Over Time: Deconstructing the Variable Foreperiod Phenomena. Journal of Experimental Psychology: Human
Perception and Performance. https://doi.org/10.1037/0096-1523. 33.6.1377

Vallesi, A., Shallice, T., \& Walsh, V. (2007). Role of the prefrontal cortex in the foreperiod effect: TMS evidence for dual mechanisms in temporal preparation. Cerebral Cortex, 17(2), 466-474. https://doi.org/ 10.1093/cercor/bhj163

Vangkilde, S., Coull, J. T., \& Bundesen, C. (2012). Great expectations: Temporal expectation modulates perceptual processing speed. Journal of Experimental Psychology: Human Perception and Performance. https://doi.org/10.1037/a0026343

Wassenberg, R., Hendriksen, J. G. M., Hurks, P. P. M., Feron, F. J. M., Keulers, E. H. H., Vles, J. S. H., \& Jolles, J. (2008). Development of inattention, impulsivity, and processing speed as measured by the $\mathrm{d} 2$ test: Results of a large cross-sectional study in children aged 7-13. Child Neuropsychology https://doi.org/10.1080/ 09297040601187940

Wittling, W. (1995). Brain Asymmetry in the Control of AutonomicPhysiologic Activity. In Brain Asymmetry.

Publisher's note Springer Nature remains neutral with regard to jurisdictional claims in published maps and institutional affiliations. 